\title{
Magnetar giant flare in NGC 253 seen by Fermi-GBM
}

\author{
E. Bissaldi, ${ }^{a, b, *}$ O. J. Roberts, ${ }^{c}$ P. Veres, ${ }^{d}$ M. G. Baring, ${ }^{e}$ M. S. Briggs, ${ }^{d, f}$ \\ C. Kouveliotou, ${ }^{g, h}$ G. Younes, ${ }^{g, h}$ S. I. Chastain, ${ }^{g, h}$ J.J. DeLaunay, ${ }^{i}$ D. Huppenkothen, ${ }^{j}$ \\ A. Tohuvavohu, ${ }^{k}$ P. N. Bhat, ${ }^{d, f}$ E. Göğüş, ${ }^{l}$ A. J. van der Horst, ${ }^{g, h}$ J. A. Kennea, ${ }^{m}$ \\ D. Kocevski, ${ }^{n}$ J. D. Linford, ${ }^{o}$ S. Guiriec,${ }^{g, h, o}$ R. Hamburg, ${ }^{d, f}$ C.A. Wilson-Hodge ${ }^{n}$ and \\ E. Burns ${ }^{q}$
}

${ }^{a}$ Dipartimento Interateneo di Fisica, Politecnico di Bari, Via E. Orabona 4, 70125 Bari, Italy

${ }^{b}$ Istituto Nazionale di Fisica Nucleare, Sezione di Bari, Via E. Orabona 4, 70125 Bari, Italy

${ }^{c}$ Universities Space and Research Association, 320 Sparkman Drive, Huntsville, AL 35805, USA

${ }^{d}$ Center for Space Plasma and Aeronomic Research, University of Alabama in Huntsville, Huntsville, AL 35899, USA

${ }^{e}$ Department of Physics and Astronomy, Rice University, Houston, TX 77251, USA

${ }^{f}$ Space Science Department, University of Alabama in Huntsville, Huntsville, AL 35899, USA

${ }^{g}$ Department of Physics, The George Washington University, 725 21st Street NW, Washington, DC 20052, USA

${ }^{h}$ Astronomy, Physics, and Statistics Institute of Sciences (APSIS), The George Washington University, Washington, DC 20052, USA

${ }^{i}$ Department of Physics, The Pennsylvania State University, University Park, PA 16802, USA

${ }^{j}$ Center for Data-Intensive Research in Astronomy and Cosmology (DIRAC), Department of Astronomy, University of Washington Seattle, WA 98195, USA

${ }^{k}$ Department of Astronomy \& Astrophysics, University of Toronto, Toronto, Ontario, Canada M5S $3 H 4$

${ }^{l}$ Sabanc University, Faculty of Engineering and Natural Sciences, Istanbul 34956, Turkey

${ }^{m}$ Department of Astronomy and Astrophysics, The Pennsylvania State University, 525 Davey Lab, University Park, PA 16802, USA

${ }^{n}$ Astrophysics Branch, ST12, NASA/Marshall Space Flight Center, Huntsville, AL 35812, USA

${ }^{\circ}$ National Radio Astronomy Observatory, P.O. Box O, Socorro, NM 87801, USA

${ }^{p}$ NASA Goddard Space Flight Center (GSFC), Greenbelt, MD 20771, USA

${ }^{q}$ Department of Physics and Astronomy, Louisiana State University, Baton Rouge, LA 70803 USA

E-mail: elisabetta.bissaldi@ba.infn.it

\footnotetext{
*Presenter
} 
Magnetar giant flares (MGFs) are enormous eruptions likely triggered by surface disruptions in magnetars, neutron stars with the strongest-known magnetic fields. Such events can be detected in both X- and gamma-ray bands, but are very rare. Almost 30 magnetars have been cataloged in our Galaxy, exhibiting occasional X-ray activity, but only two have produced giant flares to date. The most recent one, emitted by SGR 1806-20 in 2004, showed an initial very short and bright main spike, causing the saturation of the observing instruments and thus precluding reliable flux measurements.

Here we report the observation and analysis of GRB 200415A, a very short and bright GammaRay Burst detected by the Fermi Gamma-Ray Burst Monitor (GBM) as well as by several other instruments participating in the InterPlanetary Network (IPN) system, which located it in a region spatially coincident with the nearby galaxy NGC 253. Analysis of the event revealed peculiar spectral and temporal properties, which are not typically seen in GRBs: a very short rise time of the initial hard spike, strong submillisecond variability, a flat spectrum, and an unusually low isotropic energy release. Therefore we concluded that GRB 200415A is not a classical short GRB due to the merger of two binary neutron stars, but rather a MGF produced by an extragalactic magnetar.

$37^{\text {th }}$ International Cosmic Ray Conference (ICRC 2021)

July 12 th - 23rd, 2021

Online - Berlin, Germany 


\section{Introduction}

Magnetars are neutron stars that exhibt the most powerful magnetic fields known: up to a thousand times the intensity of typical neutron stars. Small disturbances to their magnetic field can cause brief "eruptions", which can emit sporadic flashes or bursts of X-rays for long periods, weeks or more. Rarely, magnetars produce huge eruptions, called flares or giant flares (GFs), characterized by an intense flash of gamma rays, followed by a more gradual tail of periodic emission. These variations result from the rotation of the magnetar, which repeatedly brings the position of the emitting region in and out of the field of view of the Earth.

To date, 29 magnetars have been cataloged in the Milky Way. Most of these galactic magnetars occasionally exhibit X-ray activity, but only two of these have produced giant flares. The most recent event, which dates back to December 2004, produced measurable changes in the Earth's atmosphere, despite the fact that it was about 30 kly away. The extreme intensity of these events has always saturated the scientific instruments that have detected them, and therefore many questions have remained unresolved. Various theories have proposed that these magnetar flares constituted a subset of the more frequent short GRBs (sGRBs). However, the sensitivity of the instruments currently operating prevented us from revealing the periodic tail of the emission, and therefore being able to obtain a conclusive observation. Furthermore, the instrumental saturation during the initial spike effectively precluded an in-depth study of the intrinsic characteristics of giant flares.

On April 15 $5^{\text {th }}, 2020$, instruments on international space missions including Fermi, Wind, Mars Odyssey and INTEGRAL, which participate in a GRB location system called the InterPlanetary Network (IPN), operational since the late 1970s, recorded a signal both in X-ray and in the gammaray band, coming from the same area of the sky, and in a very short time interval. The first instrument to be triggered at 08:48:05.563746 UT was Fermi-GBM [1]. The event, which appeared to be extremely bright, short and spectrally hard, was initially classified as a short burst, GRB 200415A [2]. Few hours later, the IPN reported a triangulation of this event [3] noting that the location box, a 17 square arc-minute region, contained the nearby Sculptor Galaxy (NGC 253), an active star-bursting intermediate spiral galaxy located $\sim 3.5 \mathrm{Mpc}$ away [5]. The IPN Team first noted the light curve shape to be very similar to previsous extragalactic soft gamma-repeater giant flare candidates, namely GRB 051103 [6] and GRB 070201 [7]. Finally, observations of this peculiar event were reported by the Swift's Burst Alert Telescope (BAT) Team which discovered it from an offline analysis making use of the Gamma-ray Urgent Archiver for Novel Opportunities (GUANO) [8] pipeline; by the Fermi Large Area Telescope (LAT) Collaboration, announcing high-energy $(>100 \mathrm{MeV})$ emission from the transient $[9,10]$; and finally by the Atmosphere-Space Interactions Monitor (ASIM) Team [11], also claiming detection of significant emission up to several MeV.

In this contribution we summarize the main aspects of the Fermi-GBM observations of GRB 200415A. For a full account please refer to [12].

\section{Temporal analysis}

We studied the temporal characteristics of GRB 200415A by analysing both the Fermi-GBM and the Swift-BAT data. Panel a), b) and c) of Fig. 1 show the corresponding lightcurves in three 


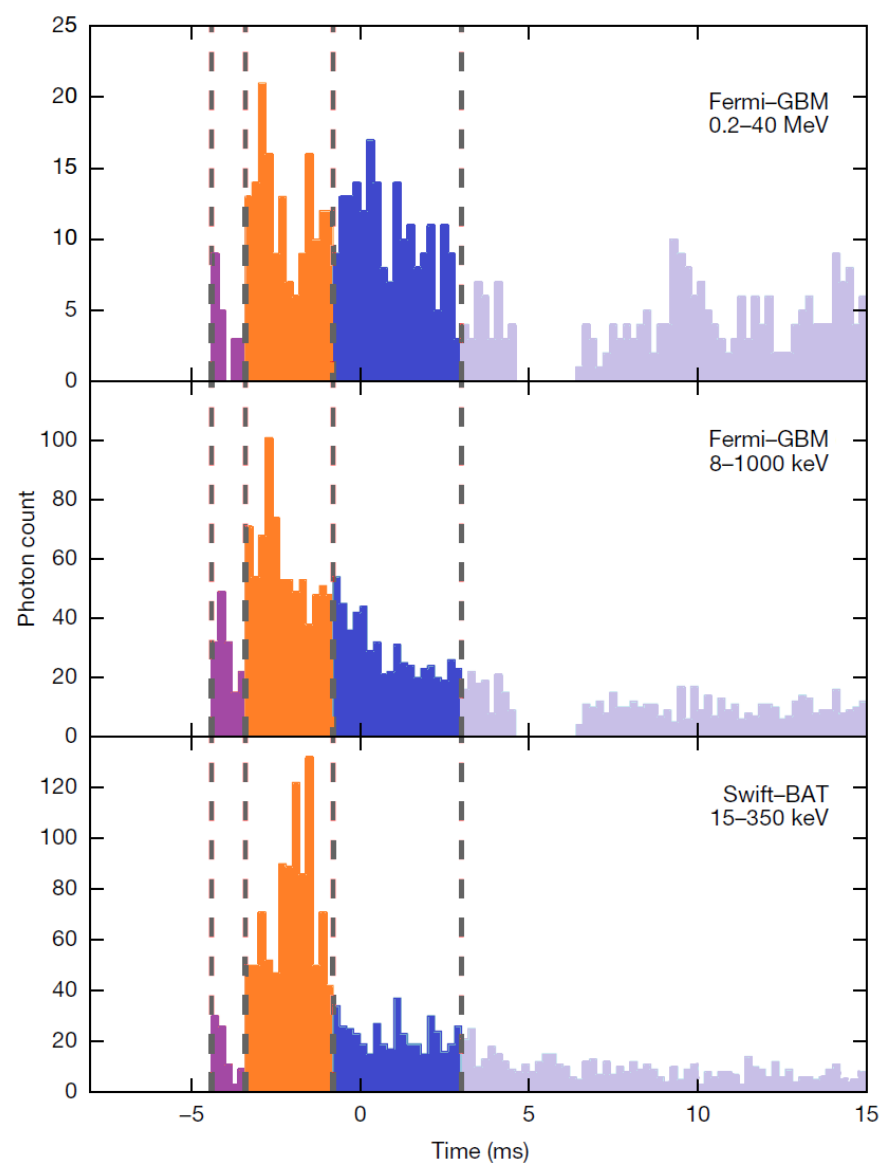

Figure 1: Lightcurves with $0.2 \mathrm{~ms}$ resolution in the $0.2-40 \mathrm{MeV}$ (Fermi-GBM, top panel), 8-1000 keV (Fermi-GBM, middle panel) and 15-350 keV (Swift, bottom panel). Dashed vertical lines divide the lightcurves in four intervals, highlighted in different colors. Adapted from [12].

energy ranges. We divided the event in four time intervals, indicated by dashed lines and different colours. High-time resolution GBM data suffered from a minor bandwidth saturation in a time interval from $T_{0}-2.4 \mathrm{~ms}$ to $T_{0}-0.8 \mathrm{~ms}$. Therefore, we used the BAT data only to determine the $\mathrm{T}_{90}$ and $\mathrm{T}_{50}$ duration of the burst, which are $140.8_{-0.6}^{+0.5} \mathrm{~ms}(1 \sigma)$ and $54.7_{-0.4}^{+0.5} \mathrm{~ms}(1 \sigma)$, respectively. We also computed the rise time of the first pulse (interval 1), defined as the time elapsed between $10 \%$ and $90 \%$ of the peak, which starts at $-4.4 \mathrm{~ms}$ and is unaffected by data saturation. We find a rise time $T_{\text {rise }}=77 \pm 23 \mu s(1 \sigma)$.

We performed a timing analysis on the GBM light curve, searching for a spin frequency in the range $0.02-50 \mathrm{~Hz}$, using an unbinned and a logarithmically binned periodogram; in particular, we searched for signals with at least $P<0.01$ (corrected for the number of frequencies and segments). We did not detect any signal that could be associated with stellar rotation. We also searched for Quasi-Periodic Oscillations (QPOs), possible signatures of seismic vibrations seen in the oscillating tails of confirmed GFs, in the $40-4000 \mathrm{~Hz}$ window and in the energy range 8-1000 $\mathrm{keV}$ using Fermi-GBM data. We found a candidate broad QPO at a frequency of $v \sim 180 \mathrm{~Hz}$ in the decaying tail (from 7 to $160 \mathrm{~ms}$ post trigger) of GRB 200415A with a significance of $\sim 2.5 \sigma$. 


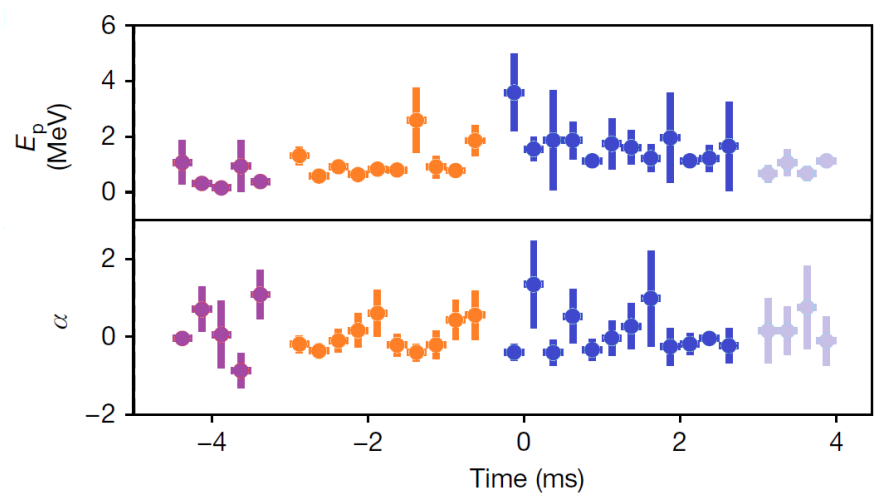

Figure 2: Evolution of the peak energy $E_{\mathrm{p}}$ (top panel) and the power-law index $\alpha$ (bottom panel) over a time interval encompassing intervals 1, 2, 3 and the onset of interval 4. The color code is the same as in Figure 1. The temporal resolution is $250 \mu \mathrm{s}$. Adapted from [12].

\section{Spectral analysis}

We performed both time-integrated and time-resolved spectral analyses of the GBM data, focusing on the sub-millisecond structures in the lightcurve. The spectral range used in our analysis was $8 \mathrm{keV}$ to $10 \mathrm{MeV}$. We fitted the differential photon number spectrum $(d N / d E)$ in all four intervals defined in the left panels of Figure 1 and found that it is best described by a power-law with an exponential cutoff, i.e. the so-called Comptonized model.

We performed a time-resolved spectral analysis on sub-millisecond timescales with a resolution of $\Delta t=250 \mu$ s over the interval shown in Figure 2, encompassing intervals 1 through 4 (defined in Figure 1). The top panel shows the evolution of the peak energy $E_{\mathrm{p}}$, which reaches its highest

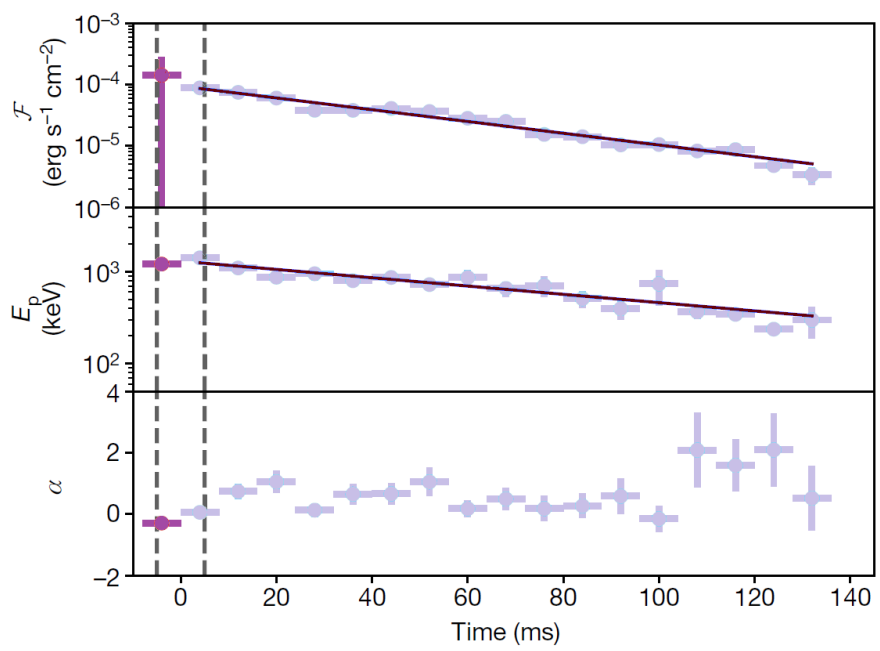

Figure 3: Evolution of the energy flux $\mathcal{F}$ (top panel), the peak energy $E_{\mathrm{p}}$ (middle panel) and the photon index $\alpha$ (bottom panel) over a time interval covering the full $T_{90}$ duration, with a temporal binning of $8 \mathrm{~ms}$. The first purple point corresponds to the first 3 intervals defined in Figure 1, while the light violet points correspond to interval 4 (tail of the burst emission). Brown lines indicate the exponential fits to $\mathcal{F}$ and $E_{\mathrm{p}}$ over the tail emission. All fit errors and error bars are at the $1 \sigma$ confidence level. Adapted from [12]. 


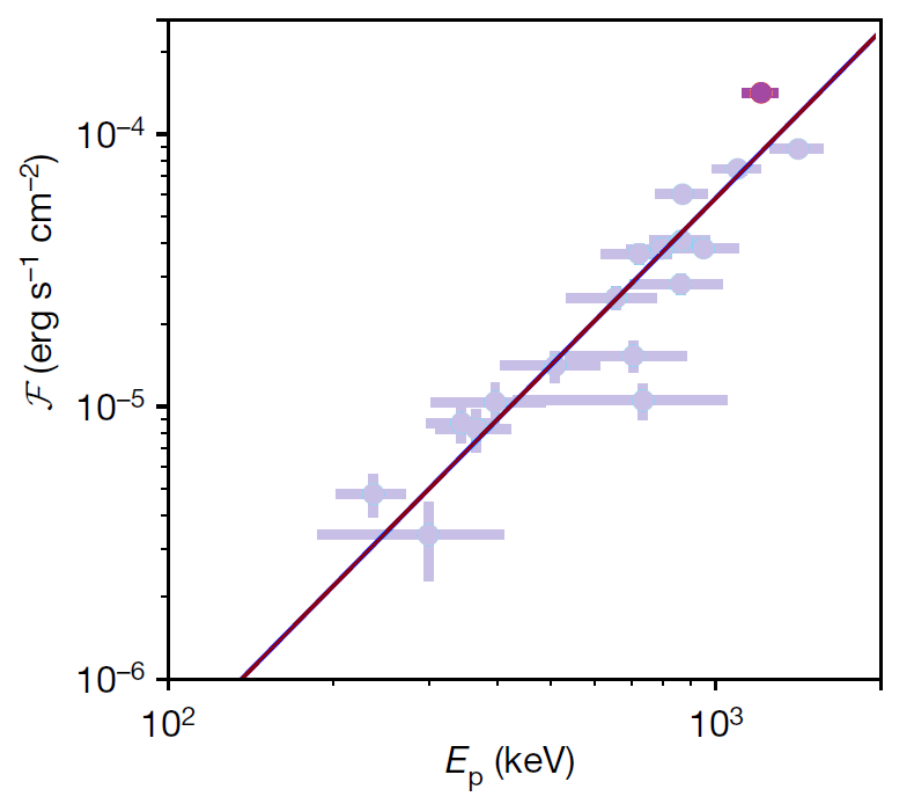

Figure 4: Relationship between the flux $\mathcal{F}$ and the peak energy $E_{\mathrm{p}}$. The brown line is a power-law fit to data relative to interval 4 . All fit errors and error bars are at the $1 \sigma$ confidence level. The color code is the same as in Figure 3. Adapted from [12].

value at the onset of interval $3\left(E_{\mathrm{p}}=1860 \pm 160 \mathrm{keV}\right.$ at $\left.T_{0} \sim-0.8 \mathrm{~ms}\right)$, while it remains relatively constant throughout most of the event. Also the photon index $\alpha$, shown in the bottom panel of Figure 2, stays relatively constant at $\alpha \sim 0$ during the event. We observed the same behaviour over the entire tail emission, as can be seen in the bottom left panel of Figure 3. This would be indeed very unusual for a short GRB.

The top and middle left panels of Figure 3 show that both the energy flux $\mathcal{F}$ and the peak energy $E_{\mathrm{p}}$ follow an exponential decay trend over interval 4 . The energy flux decay occurs on a timescale of $\tau=45 \pm 3 \mathrm{~ms}$, while the peak energy $E_{\mathrm{p}}$ is observed to decay on a longer timescale of $\tau=100 \pm 1 \mathrm{~ms}$. This exponential behaviour has been observed in other extra-galactic GF candidates [4].

Figure 4 shows the distribution of the flux versus the peak energy. The color code is the same as in Figure 3. The brown line represents a power-law fit to data of interval 4, i.e. exclusively after the main peak. We found a power-law index of $2.04 \pm 0.37$, and we interpret this interesting $\mathcal{F} \propto E_{\mathrm{p}}^{2}$ correlation as a signature of a relativistic wind.

The highest energy photons measured by Fermi-GBM which are reliably associated with GRB 200415A have energies of $\sim 3 \mathrm{MeV}$. Using time-resolved spectral analysis we computed a time-integrated isotropic equivalent energy output of $E_{\text {iso }}=(1.51 \pm 0.02) \times 10^{46} \mathrm{erg}$ and a peak isotropic luminosity of $L_{\text {iso, } \max }=(1.53 \pm 0.13) \times 10^{48} \mathrm{erg} \mathrm{s}^{-1}$. The total event luminosity is $L_{\text {iso }}=(1.07 \pm 0.17) \times 10^{47} \mathrm{erg} \mathrm{s}^{-1}$.

Finally, we searched for radio emission associated with GRB 200415A, in four observations of NGC 253 taken with the Karl G. Jansky Very Large Array (VLA), 4.3 to 51.2 days after the event trigger. No significant variable or transient emission was identified. 


\section{Discussion}

We compare our observations of GRB 200415A to previous Fermi-GBM observations of sGRBs [13]. We find that the 64-ms peak photon flux $\left(P_{64}^{\text {catalog }}=73.7 \pm 2.1\right.$ photons $\mathrm{cm}^{-2}$ $\mathrm{s}^{-1}$ ) of GRB 200415A lies at the $97.5^{t h}$ percentile of the sGRB distribution, the peak energy $\left(E_{\mathrm{p}}^{\text {catalog }}=998 \pm 45 \mathrm{keV}\right)$ at the $79^{\text {th }}$ percentile, and the photon index $\left(\alpha^{\text {catalog }}=0.39 \pm 0.09\right)$ at the $88.5^{\text {th }}$ percentile. It is similarly near the edge of the $\alpha$ distribution for the burst population detected with the Burst and Transient Source Experiment (BATSE) on the Compton Gamma-ray Observatory [14]. Consequently, we find the flat, hard spectral slope, high $E_{\mathrm{p}}$ and peak flux during the brightest $64 \mathrm{~ms}$ of GRB 200415A to be unusual for sGRBs, thus better explained as the initial spike of a magnetar GF from NGC 253. This interpretation is further motivated by similarities of the properties of this event to previously proposed extra-galactic GF candidates. A rapid rise time is another typical characteristic of a GF onset. Compared to the rise times reported in the GRB catalogs of GBM and BATSE, this rise time is considerably shorter than for any event in these samples.

Unfortunately, we could not detect the magnetar period-modulated tail in GRB 200415A, as it is likely below the detection threshold for Fermi-GBM given its distance to NGC 253 of $3.5 \mathrm{Mpc}$. For other extra-galactic GF candidates this feature is similarly undetected [4].

Using a GF interpretation, Fermi-GBM observations indicate that the MeV-band emission must come from a relativistic outflow that is initially highly opaque. The total luminosity measured from this event is orders of magnitude larger than the fiducial Eddington luminosity limit for a neutron star of solar mass. For GRB 200415A, we thus expect a relativistic wind with bulk Lorentz factor $\Gamma \gg 1$ to be present. The appearance of emission above the two-photon pair creation $\left(\gamma \gamma \longrightarrow e^{+} e^{-}\right)$ threshold of $m_{e} c^{2}=511 \mathrm{keV}$ (where $m_{e}$ is the electron mass) in GRB 200415A can be used to provide a lower bound on $\Gamma$ relative to the magnetar. The most conservative estimate corresponds to all observed GBM photons being below the $511-\mathrm{keV}$ threshold in the comoving frame of the plasma or photon gas. Thus we found $\Gamma>E_{\max } /\left(m_{e} c^{2}\right) \sim 6$. This GBM limit is consistent with the stronger constraints due to the detection of $\mathrm{GeV}$ photons by Fermi-LAT [10].

The observed correlation between the energy flux and the peak energy, $\mathcal{F} \propto E_{\mathrm{p}}^{2}$ shown in Figure 4) can be explained by relativistic Doppler boosting. We also observed that the FermiGBM spectrum is very flat. This is expected for a wind that for the most part is highly opaque to electron scattering, a so-called Compton cloud. The broad, flat spectrum of the GF may in fact be a superposition of Comptonized Wien-like spectra from different altitudes spanning a range of effective temperatures in the adiabatically cooling wind. It is worth noting that such flat $\alpha$ indices are inconsistent with synchrotron emission scenarios commonly invoked for GRB spectral interpretation [15].

\section{References}

[1] The Fermi GBM Team, GRB 200415A: Fermi GBM Final Real-time Localization. GRB Coordinates Network 27579 (2020).

[2] Bissaldi, E., et al., GRB 200415A: Fermi GBM observation. GRB Coordinates Network 27587 (2020). 
[3] Svinkin, D., et al., IPN triangulation of GRB 200415A (possible Magnetar Giant Flare in Sculptor Galaxy?). GRB Coordinates Network 27585 (2021).

[4] Svinkin, D., et al., A bright gamma-ray flare interpreted as a giant magnetar flare in NGC 253. Nature 589, 211 (2020).

[5] Rekola, R., et al., Distance to NGC 253 based on the planetary nebula luminosity function. Mon. Not. R. Astron. Soc. 361, 330 (2005).

[6] Frederiks, D. D., et al., On the possibility of identifying the short hard burst GRB 051103 with a giant flare from a soft gamma repeater in the M81 group of galaxies. Astronomy Letters 33, 19 (2007).

[7] Mazets, E. P., et al., A Giant Flare from a Soft Gamma Repeater in the Andromeda Galaxy (M31). The Astrophysical Journal 680, 545 (2008).

[8] Tohuvavohu, A., et al., Gamma-ray Urgent Archiver for Novel Opportunities (GUANO): Swift/BAT event data dumps on demand to enable sensitive sub-threshold GRB searches. The Astrophysical Journal Letters 900, 35 (2020).

[9] Omodei, N., et al., GRB 200415A: Fermi-LAT detection . GRB Coordinates Network 27586, (2020).

[10] The Fermi-LAT Collaboration. High-energy emission from a magnetar giant flare in the sculptor galaxy. Nature Astronomy 5, 385 (2021).

[11] Marisaldi, M., et al., GRB 200415A: ASIM observation. GRB Coordinates Network 27622, (2020).

[12] Roberts, O. J., et al., Rapid spectral variability of a giant flare from a magnetar in NGC 253. Nature 589, 207 (2021).

[13] von Kienlin, A., et al., The fourth Fermi-GBM gamma-ray burst catalog: A decade of data. The Astrophysical Journal 893, 46 (2020).

[14] Kaneko, Y., et al., The Complete Spectral Catalog of Bright BATSE Gamma-Ray Bursts. The Astrophysical Journal Suppl. Series 166, 298-340 (2006).

[15] Preece, R. D., et al., The Synchrotron Shock Model Confronts a "Line of Death" in the BATSE Gamma-Ray Burst Data. The Astrophysical Journal Letters 506, L23-L26 (1998). 